\section{P22 ANTI-CARBAMYLATED PROTEIN ANTIBODIES IN SYSTEMIC LUPUS ERYTHEMATOSUS: CLINICAL AND SEROLOGICAL ASSOCIATIONS} ${ }^{1,2}$ Micaela Fredi, ${ }^{1}$ Ilaria Cavazzana, ${ }^{1}$ Francesco Poiatti, ${ }^{1}$ Michele Boldrini, ${ }^{1,2}$ Silvia Piantoni,
${ }^{1}$ Rajesh Kumar, ${ }^{1,2}$ Roberta Ottaviani, ${ }^{1,2}$ Angela Tincani, ${ }^{1,2}{ }^{2}$ ranco Franceschini.
${ }^{1}$ Rheumatology and Clinical Immunology Unit, ASST Spedali Civili, Brescia; ${ }^{2}$ Dept. of Clinical
and Experimental Sciences, University of Brescia, Brescia, Italy

\subsection{6/lupus-2020-eurolupus.70}

Background/Purpose Anti-carbamylated protein antibodies (antiCarP) have been described not only in Rheumatoid arthritis but in other systemic autoimmune diseases. Recently, they have been reported in different cohorts of Systemic Lupus Erythematosus (SLE) with a prevalence of $9-28 \%{ }^{1-4}$ in patients selected with arthritis/arthralgias. Anti-CarP have been proposed as a marker of erosive arthritis in SLE. ${ }^{4}$ The aim was to assess the prevalence of anti-CarP in SLE patients from a single center cohort and their association to clinical and laboratory data.

Methods Serum anti-CarP levels were evaluated using a homemade ELISA $(\mathrm{nv}<340 \mathrm{AU} / \mathrm{ml})$. Clinical data were obtained from clinical charts.

Results Complete clinical and serological data were available for 314 consecutive patients: 85 (27\%) positive and 229 (73\%) negative. No association was found among CarP+ and arthritis/arthralgias. CarP + patients presented an earlier disease onset compared with CarP- (mean 28 \pm 11 vs32 \pm 14.7 , $\mathrm{p}=0.001)$, a trend towards a higher prevalence of xerophtalmia $(36 \%$ vs $26.5 \%, p=0.075$;OR:1.62,95\%CI:0.95-2.75) and extractable nuclear antigen positivity $(67 \%$ vs $54 \%, p=0.064$; OR:1.65,95\%CI:0.97-2.8). Interestingly, patients anti-CarP+ less frequently experienced class IV glomerulonephritis (12\%vs $21.8 \%, p=0.05$;OR:0.53,95\%CI:0.26-1.08). Fifty-six patients evaluated were treated with anti-Blys therapy and longitudinally sera were available (T0,T6,T12). At baseline anti-CarP were positive in $10(17.8 \%)$ and titre significantly decreased at T6 $(\mathrm{p}=0.006)$ and T12 $(\mathrm{p}=0.01)$. Negative seroconversion was observed in $7 / 10$ sera.

Conclusions The prevalence of anti-CarP antibodies found in our unselected cohort is in line with to what previously reported. In our hands, anti-CarP antibodies seems to identify a less severe form of SLE, with less kidney involvement and probably in overlap with Sjogren disease. Further studies are needed in order to be able to identify a possible role for this autoantibody.

\section{REFERENCES}

1. Ziegelasch M,et al.Arthritis Res and Ther 2016;18:289.

2. Nakabo S,et al I Rheumatol 2017

3. Pecani A,et al. Arthritis Res Ther 2016; 18: 276

4. Ceccarelli F,et al. Arthritis Res Ther 2018; 20:126.

\section{P23 LONGITUDINAL ANTINUCLEAR ANTIBODY (ANA) SEROCONVERSION IN SYSTEMIC LUPUS ERYTHEMATOSUS: A PROSPECTIVE STUDY OF SWEDISH CASES WITH RECENT-ONSET DISEASE}

\footnotetext{
${ }^{1}$ Martina Frodlund, ${ }^{1}$ Jonas Wetterö, ${ }^{2}$ Charlotte Dahle, ${ }^{3}$ Örjan Dahlström, ${ }^{1}$ Thomas Skogh, ${ }^{4} J$ ohan Rönnelid, ${ }^{1}$ Christopher Sjöwall. 'Rheumatology/Division of Neuro and Inflammation Sciences, Dept. of Clinical and Experimental Medicine, Linköping University, Linköping; ${ }^{2}$ Clinical Immunology/Division of Neuro and Inflammation Sciences, Dept. of Clinical and Experimental Medicine, Linköping University, Linköping; ${ }^{3}$ Swedish Institute for Disability Research, Dept. of Behavioural Sciences and Learning, Linköping University, Linköping; ${ }^{4}$ Dept. of Immunology, Genetics and Pathology, Uppsala University, Uppsala, Sweden
}

Background Immunoglobulin G (IgG) anti-nuclear antibodies (ANA) detected by indirect immunofluorescence (IF) microscopy remain a hallmark of systemic lupus erythematosus (SLE). Since it is controversial if IF-ANA status varies over time, we designed a prospective study with longitudinal follow-up of recent-onset patients with SLE.

Methods The study population consisted of 54 newly diagnosed SLE cases, all meeting the 1982 ACR and/or the 2012 SLICC criteria. Clinical follow-up data, including disease activity and organ damage, and serum were collected from SLE onset and onwards, in most cases yearly (0 to 96 months). IF-ANA on HEp-2 cells was analysed and categorized regarding staining patterns. Using an addressable laser bead assay (ALBIA; FIDIS ${ }^{\mathrm{TM}}$ Connective profile), we measured IgG-ANA fine specificities to Ro52/SSA, Ro60/SSA, La/ SSB, Sm, Sm/RNP, U1RNP, dsDNA, ribosomal P protein and histone.

Results At baseline, all patients were judged IF-ANA positive at an abnormal titre corresponding to the 95th percentile of healthy blood donors, but seven of 54 patients (13\%) lost IF-ANA-positivity over time, see figure 1. Homogenous $(46 \%)$ and speckled (31\%) were the most frequently observed staining patterns at inclusion, whereas $7 \%$ switched their pattern at least once during follow-up, see figure 1. Established associations between ANA fine-specificities and clinical data were confirmed. Levels of anti-Sm/RNP, but not of anti-dsDNA, correlated with clinical SLE disease activity (mSLEDAI-2K).

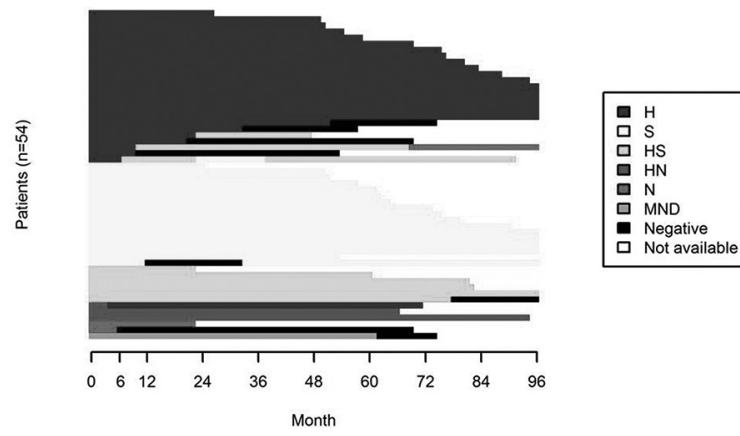

Abstract P23 Figure 1 Frequencies of ANA staining patterns over time

Conclusion A considerable proportion of Swedish patients with SLE lose IF-ANA positivity over time. Consistent staining patterns were frequent. The clinical and mechanistic relevance of ANA seroconversion remains uncertain. Further prospective evaluations in larger SLE populations with diverse ethnicities are warranted.

Acknowledgements We thank Marianne Petersson for biobank administration, all the clinicians for their efforts, and the staff at the Clinical Immunology laboratories in Linköping and Uppsala. This work was supported by grants from the Swedish Rheumatism Association, the Region Östergötland (ALF Grants), the Swedish Society of Medicine, the King Gustaf V's 80-year Anniversary foundation and the King Gustaf $\mathrm{V}$ and Queen Victoria's Freemasons foundation. 